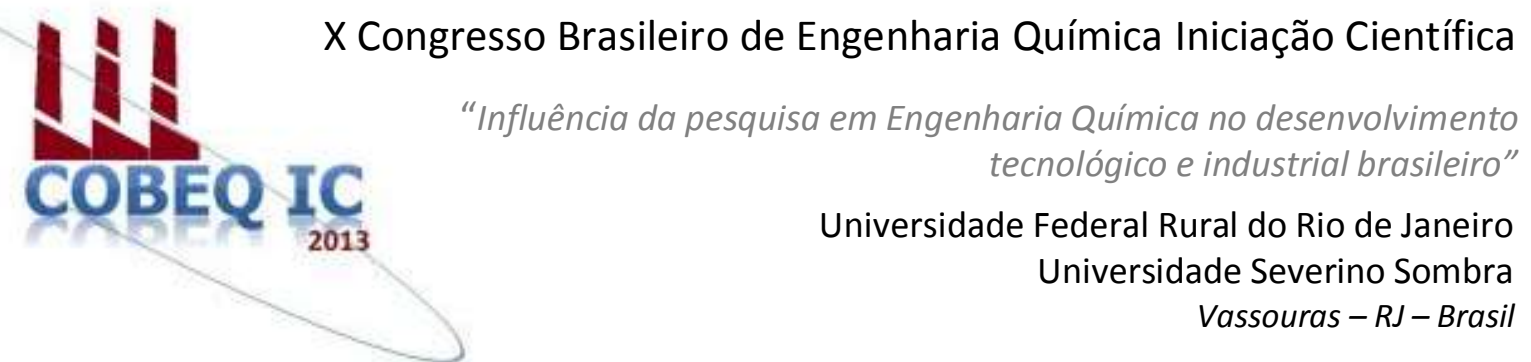

\title{
DETERMINAÇÃO DA UMIDADE DE EQUILÍBRIO DE SORÇÃO DA PIMENTA-DO-REINO
}

\author{
RAMALHO $^{* 1}$, A.P.S.; LIRA ${ }^{2}$, T.S, BACELOS ${ }^{2}$, M. S. \\ ${ }^{1}$ Aluna do DETEC/UFES ${ }^{2}$ Professor DETEC/UFES \\ Departamento de Engenharias e Tecnologia - Universidade Federal do Espírito Santo \\ Endereço - UFES/ Ceunes, Rodovia BR 101 Norte, km 60, Bairro Litorâneo, CEP. 29.932-540, RJ, \\ email:marcelo.bacelos@ufes.br
}

\begin{abstract}
RESUMO - Devido ao elevado cultivo de pimenta-do-reino no Norte Espírito Santo é necessário o estabelecimento de condições ótimas de secagem e armazenamento do produto. Para tal, é de suma importância o conhecimento das isotermas de equilíbrio destas sementes. Por isso, este trabalho teve como objetivo obter os dados de umidade de equilíbrio da pimenta-do-reino e ajustá-los aos principais modelos existentes na literatura. Através do método gravimétrico estático as isotermas foram determinadas empregando-se as temperaturas de $20,30,40,50$ e $60{ }^{\circ} \mathrm{C}$, dentro de uma ampla faixa de atividade de água $(0,112-0,834)$. Os modelos de Henderson, Henderson-Thompson, Chen-Clayton e Halsey modificado foram testados para ajuste dos dados experimentais utilizando análise de regressão não-linear. As isotermas de adsorção exibiram comportamento do tipo II. Também, verificou-se que não houve efeito significativo da temperatura sobre os dados de umidade de equilíbrio. Com base na análise dos dados, verificou-se que o modelo de Chen-Clayton se ajustou melhor aos dados experimentais e o calor isostérico de adsorção da pimenta-do-reino diminuiu com o aumento da umidade de equilíbrio. Observou-se que o processo de adsorção é espontâneo para umidades acima de 0,08 .
\end{abstract}

Palavras chave: secagem, regressão não linear, método gravimétrico.

\section{INTRODUÇÃO}

A pimenta-do-reino, Piper Nigrum, é uma planta nativa da Costa do Malabar, Índia, e seu fruto é uma famosa especiaria relatada desde quatro séculos A.C. em muitas partes do mundo. No mercado mundial de pimenta-doreino, o Brasil ocupa posição de destaque, sendo responsável por aproximadamente $15 \%$ da produção do mundo. O Espírito Santo é o segundo maior produtor nacional, com desta- que para a região norte do estado que é considerada um pólo tradicional de pimentado-reino. A cada ano, são produzidas 7,5 toneladas de pimenta-do-reino no estado, produto de exportação para todo o Brasil, além de Argentina, Europa e Estados Unidos. São Mateus representa $73,5 \%$ dessa produtividade, sendo o $1^{\mathrm{o}}$ município no ranking de produção nacional. A pimenta-do-reino é uma especiaria muito apreciada na culinária de diversos paises, sendo usada como condimento e em in- 
dústrias de carnes e conservas.

Para o armazenamento de grãos adequado, parte da água contida no grão, após a colheita, precisa ser removida. Ao diminuir o teor de água de um produto, reduz-se o risco de proliferação por microorganismos e a ocorrência de reações enzimáticas, preservando assim a qualidade e o valor nutritivo do mesmo. A secagem é uma das etapas mais importantes dentro do processamento de grãos, pois remove o excesso de água de forma a propiciar condições adequadas para o beneficiamento, armazenamento e comercialização do produto.

Com respeito à secagem de pimentado-reino, estudos sobre isotermas de equilíbrio de sorção e dessorção desempenham um papel importante sobre a qualidade do produto. Isso se deve ao fato de que por meio do conhecimento destas obtêm-se as condições ótimas do processo de secagem e estocagem.

$\mathrm{Na}$ literatura, vários trabalhos têm sido realizados na obtenção de dados de umidade e equilíbrio de diversos alimentos, analisando a adequação dos modelos semi-empíricos por meio do coeficiente de correlação (Leitão, 1983, para pimenta-do-reino; Barrozo, 1995, para grãos de soja; Kurozawa et al., 2005, para cogumelo do sol). Contudo, somente poucas pesquisas têm analisado a adequação dos modelos de isotermas de equilíbrio usando as técnicas não lineares de análise de parâ-metros tais como as medidas de curvatura in-trínseca (IN) e paramétrica (PE) propostas por Bates e Watts, além do vício de Box.

Além disso, propriedades termodinâmicas, como o calor isostérico de sorção e entropia de sorção podem ser obtidos a partir das isotermas de sorção a diferentes temperaturas. $\mathrm{O}$ conhecimento do calor isostérico em função do teor de água de equilíbrio é essencial nos estudos de secagem $\mathrm{e}$ armazenamento de produtos agrícolas servindo para estimar as necessidades energéticas do proces-so de secagem além de fornecer dados sobre o estado da água no produto.

Ante o exposto, o objetivo deste trabalho é determinar as isotermas de sorção da pimenta-do-reino e obter os valores do calor isostérico de sorção em função da umidade de equilíbrio.

\section{METODOLOGIA}

\section{Materiais}

O material estudado é a pimenta-doreino (Piper Nigrum) negra pós-colheita. As amostras utilizadas são provenientes do processo de secagem ao sol. Na obtenção das isotermas de equilíbrio, as amostras são submetidas a 24 horas em estufa a $105^{\circ} \mathrm{C}$, para que depois aproximadamente $2 \mathrm{~g}$ de massa seca de pimenta-do-reino seja disposto em recipientes de polietileno. Tais amostras são submetidas a ambientes com umidade relativa controlada através do uso de um conjuto de soluções salinas específicas, conforme é mostrado no próximo item.

\section{Método gravimétrico}

O método gravimétrico estático (Rizvi, 1995) com uso de soluções salinas suturadas é empregado para determinar a umidade de equilíbrio das sementes de pimenta-do-reino secas em 20, 30, 40, 45 e $50^{\circ} \mathrm{C}$. Os sais $(\mathrm{KOH}, \mathrm{LiCl}, \mathrm{CH} 3 \mathrm{COOK}, \mathrm{MgCl} 2, \mathrm{~K} 2 \mathrm{CO} 3$, $\mathrm{NaNO} 2, \mathrm{NaCl}$ e $\mathrm{KCl}$ ) são escolhidos com base no trabalho de Barrozo (1995). Estes permitem que as amostras sejam submetidas uma ampla faixa de umidade relativa de 11,2 a $83,4 \%$.

\section{Procedimento experimental}

As amostras de pimenta-do-reino são dispostas em recipientes de polietileno (copo plástico) e acondicionadas em frascos de vidro hermeticamente fechados, de $6,5 \mathrm{~cm}$ de altura e $6 \mathrm{~cm}$ de diâmetro interno, contendo as soluções salinas saturadas.

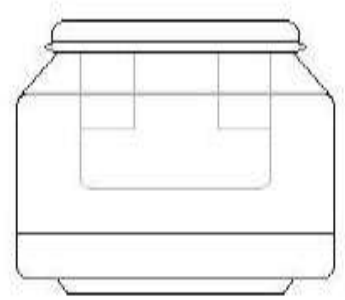

Figura 1 - Modelo de frasco e suporte de polietileno utilizados

Todos os ensaios são realizados em triplicata nas temperaturas de $20,30,40,50$ e $60^{\circ} \mathrm{C}$. O processo é acompanhado por meio da medida de massa das amostras a cada três dias, até que o equilíbrio seja estabelecido. $\mathrm{O}$ equilíbrio é comprovado através de três medidas 
consecutivas, quando verifica-se que a massa das amostras não se altera significativamente.

\section{Modelos de isotermas de equilíbrio}

Baseando-se na literatura, os principais modelos semi-empíricos são escolhidos para análise dos dados experimentais. A Tabela mostra os modelos testados para prever os dados de umidade de equilíbrio de em função da umidade relativa e da temperatura.

Tabela 1. Modelos Semi Empíricos

\begin{tabular}{|l|l|}
\hline Equação & Referência \\
\hline $\mathbf{M}_{\mathrm{eq}}=\left[\frac{\ln (1-\mathbf{U R})}{-\mathbf{a T} \mathbf{T}_{\mathrm{s}}}\right]^{1 / 6}$ & Henderson (1952) \\
\hline $\mathbf{M}_{\mathrm{eq}}=\left[\frac{\ln (1-\mathbf{U R})}{-\mathbf{a}\left(\mathbf{T}_{\mathrm{s}}+\mathbf{c}\right)}\right]^{1 / 6}$ & Thompson (1968) \\
\hline $\mathbf{M}_{\mathrm{eq}}=\frac{-1}{\mathbf{c T} \mathbf{T}_{\mathrm{s}}{ }^{d}} \ln \left(\frac{\ln (\mathbf{U R})}{-\mathbf{a} \mathbf{T}_{\mathrm{s}}{ }^{\mathrm{b}}}\right)$ & $\begin{array}{l}\text { Chen e Clayton } \\
(1971)\end{array}$ \\
\hline $\mathbf{M}_{\mathrm{eq}}=\left[\frac{\exp \left(\mathbf{a}-\mathbf{c} \cdot \mathbf{T}_{\mathbf{s}}\right)}{-\ln (\mathbf{U R})}\right]^{1 / 1 /}$ & Osborn et al (1989) \\
\hline
\end{tabular}

Em que Meq é a umidade de equilíbrio do material (base seca), Ts é a temperatura do material, UR é a umidade relativa do ar e a, b e c são parâmetros de ajuste.

\section{Análise estatística}

A adequação dos modelos semiempíricos é realizada por meio de técnicas de regressão não lineares, tais como: medidas de curvaturas propostas por Bates e Watts as quais são característica do modelo (intrínseca) ou do parâmetro (paramétrica) e vício de Box. Após ajuste dos modelos empíricos aos dados experimentais, as estimativas dos parâmetros são validadas estatisticamente com base em medidas de não linearidade. Para tanto, são analisados os vícios de Box e as medidas de curvatura de Bates e Watts, além das inferências estatísticas razão $\mathrm{F}$ e coeficiente de determinação R2.

\section{Cálculo do calor isostérico e entropia de sorção}

Para a determinação do calor isostérico $\left(\mathrm{q}_{\mathrm{st}}\right)$ e da entropia de sorção $(\Delta \mathrm{S})$ da umidade contida no material para diferentes valores de temperatura e umidade relativa, utilizou-se o mesmo procedimento utilizado por Arrieche et al.(2012). A Tabela 2 apresenta o equacionamento utilizado para determinar o calor isotérico e entropia de sorção.

O calor de sorção para cada conteúdo de umidade das partículas foi derivado da equação de Clausius-Clapeyron (1). Considerando que o calor de sorção é independente da temperatura e integrando a Equação 1, obtém-se a Equação 2.

Portanto, conhecendo a relação entre a umidade relativa e $\mathrm{a}_{\mathrm{w}}$ e construindo um gráfico de $\ln \left(\mathrm{a}_{\mathrm{w}}\right)$ em função de $1 / \mathrm{T}$, pode-se de maneira prática obter o valor de $\mathrm{q}_{\mathrm{st}}$, através do coeficiente angular de uma reta obtida via regressão linear, uma vez que o valor de $\mathrm{R}$ é conhecido.

Tabela 2. Equações para o cálculo do calor isostérico e entropia de sorção

$\left.\frac{\partial \ln \left(a_{w}\right)}{\partial(1 / T)}\right|_{X}=-\frac{Q s-\lambda}{R}=-\frac{q_{s}}{R}$
$\ln \left(a_{w}\right)=-\frac{q_{s}}{R}\left(\frac{1}{T}\right)+C$
$\Delta \mathrm{S}=\frac{\Delta \mathrm{H}-\Delta \mathrm{G}}{\mathrm{T}}$
$\Delta G=R T \ln a_{w}$
$\ln a_{w}=\frac{\Delta H}{R T}-\frac{\Delta S}{R}$

O calor isostérico de sorção também pode ser obtido a partir de outra abordagem. A variação da entropia diferencial molar pode ser calculada pela equação de Gibbs-Helmholtz (3). Onde a energia livre é calculada por (4). Substituindo a Equação (3) na Equação (4) e rearranjando, obtém-se a Equação (5) que é similar à Equação (2), mas nesta forma com o termo referente à entropia de sorção $(\Delta S)$ explícito.

\section{RESULTADOS E DISCUSSÕES}

A Figura 2 apresenta a umidade de equilíbrio em função da atividade de água, parametrizada nas temperaturas de $20^{\circ}, 30^{\circ}$, $40^{\circ}, 50^{\circ}$ e $60^{\circ}$ C. Também, observa-se na figura 2 que as isotermas de adsorção da pimenta-do-reino são do tipo II, de acordo com a classificação de Brunauer (Brunauer et al., 
1938), este tipo é apresentado por isotermas de produtos orgânicos.

Analisando-se a Figura 2, nota-se que a umidade de equilíbrio aumenta com o acréscimo da atividade de atividade de água. Para a faixa de temperatura empregada $\left(20^{\circ}\right.$ a $60^{\circ} \mathrm{C}$ ), observa-se, também, que não há efeito significativo da temperatura sobre a umidade de equilíbrio. Resultados semelhantes são obtidos por Silva et al.( 2010), para isotermas de adsorção do coentro desidratado e, também, por Alcântara et al.(2008), para isotermas de adsorção do pedúnculo seco de caju.

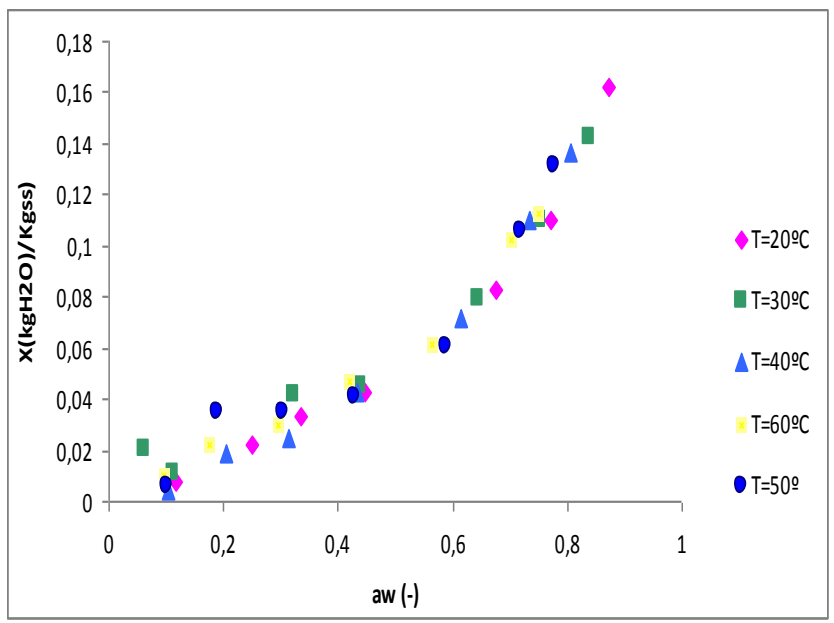

Figura 2, umidade de equilíbrio em função das temperaturas de $20,30,40,50$ e $60^{\circ}$

De acordo com Ferreira \& Pena (2003), esse comportamento pode ser provavelmente explicado através de dois mecanismos: 1) o aumento da temperatura pode provocar modificações na estrutura física do produto, disponibilizando um número maior de sítios ativos com afinidade por moléculas de água; ou 2) pode provocar um aumento na solubilidade de solutos intrínsecos ao produto, fazendo com que um número maior de moléculas de água fique retido na monocamada.

Observa-se na Tabela 3 que de acordo com os resultados dos valores do coeficiente de determinação (R2 ), o modelo de ChenClayton descreveu melhor as isotermas de adsorção da pimenta-do-reino, nas cinco temperaturas estudadas. Comparando-se os resultados das medidas de curvatura (IN) com o valor $1 / 2 \sqrt{F}$, nota-se que na maioria das equações a curvatura intrínseca não foi significativa $(\mathrm{IN}<1 / 2 \sqrt{\mathrm{F}}$ ). Isto indica que a não linearidade do "locus de solução" foi pequena. Com relação a curvatura paramétrica (PE), em todas as equações essa medida foi significativa $(\mathrm{PE}>1 / 2 \sqrt{\mathrm{F}})$, demonstrando então que pelo menos um dos parâmetros, de cada uma dessas equações, apresenta um forte comportamento não linear. Além disso, os parâmetros de todas as equações apresentaram altos vícios de Box, também, indicando comportamento não linearidade significativo.

Comprova-se que a equação de ChenClayton, além de apresentar alto $\mathrm{R}^{2}$, demonstra também, o comportamento mais próximo da normalidade na distribuição de parâmetros, onde apenas o parâmetro "a" apresentou um vício elevado, confirmando a validade dos resultados inferenciais dos estimadores de mínimos quadrados para essa equação.

A Figura 3 mostra os dados de $\ln \left(\mathrm{a}_{\mathrm{w}}\right)$ em função do inverso da Temperatura (1/T) e da umidade de equilíbrio (Xeq). Com base na análise dos dados, ajustadas pelo modelo de Chen-Clayton, nota-se decréscimo do calor isostérico de sorção $\left(\mathrm{Q}_{\mathrm{ST}}\right)$ com o aumento da umidade de equilíbrio.

Resultados e tendências semelhantes foram encontrados por e Silva et al. (2002) ao estudarem isotermas e o calor isostérico da palma forrageira enriquecida proteicamente e polpa de manga, respectivamente. Observa-se que quanto menor a umidade de equilíbrio das sementes, mais energia é requerida para evaporar a água ligada à estrutura biológica do produto. Dessa maneira, a evaporação de água da pimenta-do-reino requer energia para superar o calor de evaporação da água pura.

A determinação de $\Delta S$ é importante, pois, com o este resultado juntamente com $\mathrm{q}_{\mathrm{st}}$ (Callen, 1985) pode-se calcular a energia livre de Gibbs $(\Delta \mathrm{G})$. Esta função termodinâmica de estado representa a quantidade máxima de energia liberada em um processo ocorrendo à temperatura e pressão constantes.

Também, a entropia pode ser usada como indicador da espontaneidade do processo de adsorção.

A Tabela 4 apresenta os valores de entropia do processo. Para o intervalo de $0,04<$ Xeq $<0,08$, os valores de variação de entropia sugerem que o processo de adsorção 
Tabela 3. Medidas de não linearidade para os modelos de umidade de equílibrio semiempíricos.

\begin{tabular}{|c|c|c|c|c|c|c|c|}
\hline Equação & $\begin{array}{c}\text { Coeficient } \\
\text { e de } \\
\text { Correlaçã } \\
\text { o } \\
\end{array}$ & $\begin{array}{l}\text { Curvatura } \\
\text { Intrínseca }\end{array}$ & $\begin{array}{c}\text { Curvatura } \\
\text { Paramétri } \\
\text { ca }\end{array}$ & Parâmetro & $\begin{array}{c}\text { Valor } \\
\text { Estimado }\end{array}$ & $\begin{array}{l}\text { Desvio } \\
\text { Padrão }\end{array}$ & \% Vício \\
\hline \multirow[t]{2}{*}{ Henderson } & \multirow[t]{2}{*}{0,72972} & \multirow[t]{2}{*}{0,2783} & \multirow[t]{2}{*}{10,0996} & $\mathrm{~A}$ & 2,1393 & 1,107 & 117,8708 \\
\hline & & & & $\mathrm{B}$ & 1,7215 & 0,2171 & 8,027 \\
\hline \multirow{3}{*}{$\begin{array}{l}\text { Henderson- } \\
\text { Thompson }\end{array}$} & \multirow[t]{3}{*}{0,9561} & \multirow[t]{3}{*}{2,8357} & \multirow[t]{3}{*}{278381,2} & $\bar{A}$ & 0,0001 & 0,0004 & 31221,73 \\
\hline & & & & $\mathrm{B}$ & 1,5864 & 0,0784 & 308,0386 \\
\hline & & & & $\bar{C}$ & 3593,56 & $\begin{array}{c}27451,285 \\
9\end{array}$ & 9393653 \\
\hline \multirow{4}{*}{$\begin{array}{l}\text { Chen- } \\
\text { Clayton }\end{array}$} & \multirow[t]{4}{*}{0,9538} & \multirow[t]{4}{*}{0,1553} & \multirow[t]{4}{*}{16,126} & $\bar{A}$ & 1,4973 & 0,7802 & 25,3451 \\
\hline & & & & $\mathrm{B}$ & 0,1168 & 0,1438 & $-1,5392$ \\
\hline & & & & $\mathrm{C}$ & 16,2069 & 5,8092 & 12 \\
\hline & & & & $\mathrm{D}$ & 0,0351 & $-0,1698$ & $-1,4341$ \\
\hline \multirow{3}{*}{$\begin{array}{c}\text { Halsey } \\
\text { modificada }\end{array}$} & \multirow[t]{3}{*}{0,9595} & \multirow[t]{3}{*}{1,3993} & \multirow[t]{3}{*}{4,6162} & $\bar{A}$ & 0,0067 & 0,0019 & $-232,7199$ \\
\hline & & & & $\bar{B}$ & 1,2143 & 0,0578 & 128,9094 \\
\hline & & & & $\mathrm{C}$ & $-4,2076$ & 0,131 & 86,1493 \\
\hline
\end{tabular}

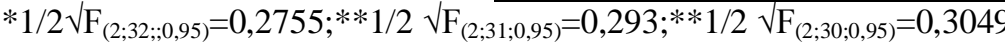

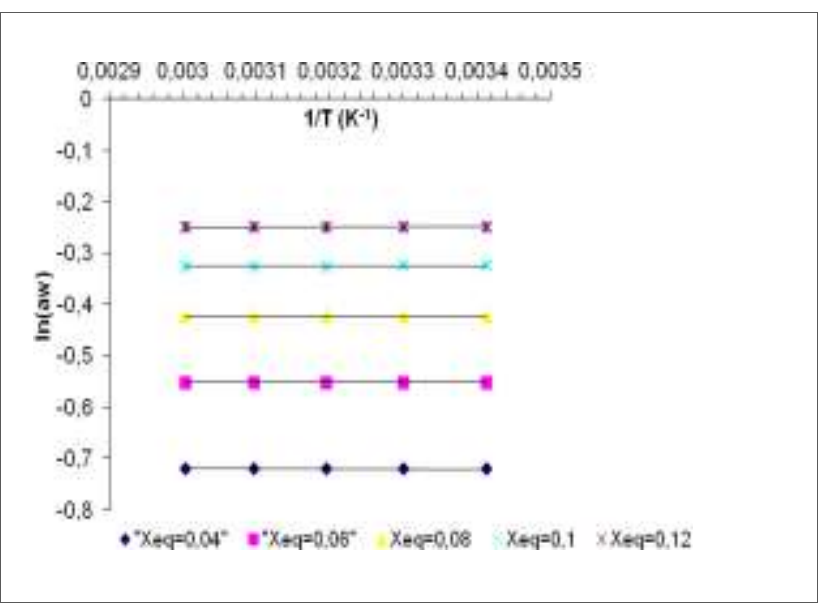

Figura 3 - Ln (aw) em função de 1/T e Xeq.

Tabela 4. Valores de calor isostérico e entropia de sorção

\begin{tabular}{|l|l|l|}
\hline Xeq & Qst $(\mathbf{K j} / \mathbf{m o l})$ & Entropia \\
\hline 0,04 & 1,6126 & $-13,44908$ \\
\hline 0,06 & 1,1575 & $-9,65355$ \\
\hline 0,08 & 0,2779 & $-2,317686$ \\
\hline 0,1 & $-1,242$ & 10,35828 \\
\hline 0,12 & $-3,7096$ & 30,93806 \\
\hline
\end{tabular}

entropia sugerem que o processo de adsorção não é espontâneo $(\Delta \mathrm{S}<0)$. Entretanto, para
$0,1<\mathrm{Xeq}<0,12$, a adsorção é espontânea $(\Delta \mathrm{S}>0)$.

\section{CONCLUSÕES}

Com base nas análises estabelecidas neste trabalho pode-se concluir que:

- o método gravimétrico, com o uso de soluções salinas se mostrou adequado para determinar a umidade de equilíbrio da pimenta-do-reino;

- o modelo de Chen-Clayton representou melhor os dados de umidade de equilibrio de sorção da pimenta do reino

- o calor isostérico de sorção, determinado pela equação de Clausius-Clapeyron, diminuiu com o aumento da umidade de equilíbrio das amostras;

- nas condições operacionais empregadas, observou-se a adsorção torna-se espontânea para Xeq >0,08.

\section{Nomenclatura}

a,b,c,d parâmetros de ajuste adimensionais aw atividade de água

Qst calor isostérico de adsorção (kJ. mol $\left.{ }^{-1}\right)$

$\mathrm{R}$ constante universal dos gases $\left(8,314 \mathrm{~J} \mathrm{~mol}^{-}\right.$ ${ }^{1} . \mathrm{K}^{-1}$ ) 
b.s. base seca $(\%)$

aw atividade de água

C constante de integração

$\mathrm{Q}_{\mathrm{s}}$ calor isostérico de sorção $\left(\mathrm{kJ} . \mathrm{mol}^{-1}\right)$

$\mathrm{Q}_{\mathrm{s}}$ calor total de sorção $\quad\left(\mathrm{kJ} \cdot \mathrm{mol}^{-1}\right)$

$\mathrm{R}$ constante universal dos gases (J.mol-1. $\mathrm{K}^{-1}$ )

$\mathrm{R}^{2}$ coeficiente de correlação do ajuste

T temperatura $(\mathrm{K})$ ou $\left({ }^{0} \mathrm{C}\right)$

UR umidade relativa do ar

Xeq umidade de equilíbrio

$\lambda$ calor latente da água pura $\left(\mathrm{kJ} \cdot \mathrm{mol}^{-1}\right)$

$\Delta \mathrm{G}$ variação da energia livre de Gibbs (kJ.mol ${ }^{-}$ $\left.{ }^{1}\right)$

$\Delta \mathrm{H}$ variação da entalpia $\left(\mathrm{kJ} . \mathrm{mol}^{-1}\right)$

$\Delta \mathrm{S}$ variação da entropia de sorção $\left(\mathrm{kJ}^{\mathrm{m}} \cdot \mathrm{mol}^{-1} \mathrm{~K}^{-}\right.$ $\left.{ }^{1}\right)$

\section{REFERÊNCIAS BIBLIOGRÁFICAS}

ALCÂNTARA S.R., ALMEIDA, F.A. C., Da Silva, F.L. H., GOMES, J.P. (2009), Isotermas de adsorção do pedúnculo seco do caju, Revista Brasileira de Engenharia Agrícola e Ambiental, v.13, n.1, PB, UAEA/UFCG, Campina Grande, 81-87 p.

ARRIECHE, L.S., PICELLI, R.M., SRTORI, D.J.M (2012), Análise das Energias Envolvidas no Processor de Dessorção de Umidade em Sementes Silvestres, Revista de Ciências Exatas, RJ, EDUR, v 27/31, n.2, jul-dez, 07-25 p.

BARROZO, M.A.S. (1995), Transferência de Calor e Massa entre o ar e Sementes de Soja em Leito Deslizante e Escoamentos Cruzados, Tese (Doutorado em Engenharia Química), Programa de PósGraduação em Engenharia Química, Univer-sidade Federal de São Carlos, São Carlos.

BRUNAUER, S.; EMMETT, P. H.; TELLER, E.(1938), Adsorption of gases in multimolecular layer. Journal of American Chemical Society, v.60, n. 02,309-319 p.

CALLEN, H.B. Thermodynamics and an introduction to thermostatistics. (1985), 2nd Edition, New York - NY, John Wiley, $493 \mathrm{p}$.

CHEN, C.S.; CLAYTON, J.T. (1971), The effect of temperature on sorption isotherms of biological materials. Transactions of the ASAE, v. 14, 927-929 p.
CHU, S.T. e HUSTRULID, A. (1968), Numerical solution of difusion equations. Transactions os ASAE, Vol 11, 705-709 p.

FERREIRA, C. D.; PENA, R. S. (2003), Comportamento higroscópico da farinha de pupunha (Bactris gasipaes). Ciência e Tecnologia de Alimentos, v.23, n.2, 251$255 \mathrm{p}$.

HENDERSON, S.M. (1952), A basic concept of equilibrium moisture content. Agricultural Engeneering, Vol.33 (2), 29-31 p.

KUROZAWA, L. E., EL-AOUAR, A.A, MURR, F.E.X (2005), Obtenção de Isotermas de Dessorção de Cogumelo in natura e Desidratado Osmoticamente, Departamento de Engenharia de Alimentos, Universidade Estadual de Campinas, Campinas.

LEITÃO, A.M., Algumas propriedades físicas e mecânicas da pimenta-do-reino preta (Piper nigrum L.) variedade Kalluvally (1983), Tese (Mestrado em Tecnologia de alimentos) - Faculdade de Engenharia de Alimentos e Agrícola, Universidade Estadual de Campinas, Campinas.

OSBORN, G.S., WHITE, G.M., SULAIMAN, A.H. E, WELTON L.R. (1989), Predicting Equilibrium moisture proportions of soybeans. Trasactions of ASAE, Vol. 32 (6), 2109-2113 p..

RIZVI, S.S.H. Thermodynamic properties of foods in dehydration, 1995, In: M.A. Rao, \& S.S.H. Rizvi (eds.), Engineering properties of foods. New York: Marcel Dekker, 223-309 p.

SILVA, A.S. , CARDOSO, F.A.C., ALVES, N. M.C., MELO, K.S., GOMES, J. P. (2010), Característica higroscópica e termodinâmica do coentro desidratado, v. 41, n. 2, abr-jun, Centro de Ciências Agrárias - Universidade Federal do Ceará, Fortaleza, CE, 237-244 p.

THOMPSON, L.T., PEART, R.M. e FOSTER, G.H. (1968), Mathematical Simulation of Corn Drying - a new Model. Trasactions of the ASAE, Vol. 11, 582$586 \mathrm{p}$. 